\title{
RNF185-AS1 Promotes Hepatocellular Carcinoma Progression Through Targeting miR-221-5p/integrin B5 Axis
}

\section{Chunmei Huang}

The Central Hospital of Wuhan

Ke Li

Wuhan No.1 Hospital

\section{Rongfu Huang}

the Seond Affiliated Hospital, Fujian Medical College

Jianhua Zhu ( $D$ zhujh621@163.com )

Wuhan No.1 Hospital https://orcid.org/0000-0001-7746-4100

Jiayao Yang

Wuhan No.1 Hospital

\section{Primary research}

Keywords: hepatocellular carcinoma, RNF185-AS1, miR-221-5p, integrin $\beta 5$

Posted Date: August 25th, 2020

DOl: https://doi.org/10.21203/rs.3.rs-62225/v1

License: (9) This work is licensed under a Creative Commons Attribution 4.0 International License. Read Full License

Version of Record: A version of this preprint was published at Life Sciences on February 1st, 2021. See the published version at https://doi.org/10.1016/j.lfs.2020.118928. 


\section{Abstract}

Background: Recently, long noncoding RNAs (IncRNAs) have been reported to play important role in pathogenesis of various cancers. However, the function of RNF185-AS1 in hepatocellular carcinoma (HCC) metastasis has not been well investigated. The present study aims to explore the role and mechanism of RNF185-AS1 in hepatocellular carcinoma metastasis.

Methods: The RNF185-AS1 expression in HCC cells and tissues was measured by quantitative real-time polymerase chain reaction (qRT-PCR). The functional effects of RNF185-AS1 on tumor cell proliferation, migration, invasion and epithelial-mesenchymal transition (EMT) were assessed by Cell Counting Kit-8 (CCK8) assay, colony formation assay, transwell assay and Western blot. The luciferase reporters assay, RNA-binding protein immunoprecipitation assay, qRT-PCR and Western blot were performed to explore and confirm the interaction between RNF185-AS1 and miR-221-5p and integrin $\beta 5$. The role of RNF185AS1 in tumor progression was explored through in vivo experiments.

Results: RNF185-AS1 was highly expressed in HCC tissues and cell lines. High levels of RNF185-AS1 was correlated with advanced TNM stage, distant metastasis and a poorer overall survival rate. RNF185-AS1 knockdown inhibited cell proliferation, migration, invasion and EMT. Additionally, RNF185-AS1 acted as a sponge for miR-221-5p and integrin $\beta 5$ was identified as a target gene of miR-221-5p. Rescue assays showed that miR-221-5p inhibitor or integrin $\beta 5$ overexpression rescued the function of RNF185-AS1 knockdown on cell proliferation, migration, invasion and EMT. Moreover, we found that RNF185-AS1 knockdown inhibited tumor metastases in xenograft tumor mouse model.

Conclusion: Our findings demonstrated that RNF185-AS1 promoted cell EMT and migration by regulating miR-221-5p/integrin $\beta 5$ axis in HCC.

\section{Background}

Hepatocellular carcinoma (HCC) is one of the most common malignant tumors. Although the progress in the treatment of $\mathrm{HCC}$ has been made, high rate of metastasis and tumor recurrence is the main reason for the poor prognosis of HCC patients [1]. Therefore, it is crucial to explore the molecular mechanisms in $\mathrm{HCC}$ recurrence and metastasis, so as to improve the treatment for HCC.

Long noncoding RNAs (IncRNAs) are a group of noncoding RNAs with more than 200 nucleotides in length and are without protein-coding capacity [2]. LncRNA plays an important role in regulating various biological processes of tumor cell, including proliferation, differentiation, apoptosis, migration and invasion [3-5]. Recently, accumulative evidences have demonstrated the critical involvements of IncRNAs in the development of HCC. For example, LncRNA MNX1-AS1 promotes HCC proliferation and invasion by targeting miR-218-5p/COMMD8 axis [6]. LncRNA DDX11-AS1 facilitates HCC tumor formation by interacting with EZH2/DNMT1 [7]. LINC00339 promote HCC cell proliferation and migration by sponging $\operatorname{miR}-152[8]$. 
LncRNA RNF185-AS1, located in chromosome 22, is a novel Inc RNA. To date, no evidence defined the functions of RNF185-AS1. In the present study, we searched GEPIA data and found that RNF185-AS1 was significantly upregulated in HCC patients serum. High level of RNF185-AS1 was also identified in HCC cells. RNF185-AS1 promoted HCC cells EMT and migration. Moreover, we revealed that RNF185-AS1 exerted oncogenic properties through regulating miR-221-5p/INTEGRIN B5.

\section{Materials And Methods}

\section{Human clinical samples}

$60 \mathrm{HCC}$ patients tumor tissues, adjacent normal tissues, serum samples and health control serum at Wuhan No.1 Hospital. Affiliated to Huazhong University of Science and Technology. This study was approved by the ethics committee of our hospital. All patients wrote the informed consents.

\section{Cell culture and transfection}

HCC cell lines (SMMC-7721, HepG2, HCCLM3 and Huh7) and normal hepatic cell line H7702 were purchased from the Cell Bank of the Chinese Academy of Sciences (Shanghai, China). Cell were cultured in DMEM medium (Invitrogen, Carlsbad, CA, USA) supplemented with 10\% fetal bovine serum (FBS, Invitrogen), $100 \mathrm{IU} / \mathrm{mL}$ penicillin, and $100 \mathrm{mg} / \mathrm{mL}$ streptomycin in a humidified atmosphere containing $5 \% \mathrm{CO}_{2}$ at $37^{\circ} \mathrm{C}$.

For knocking down RNF185-AS1, small interfering RNAs (siRNAs) targeting RNF185-AS1 (5'GCAGTATTCTCGGCTGTAT-3') and negative control siRNA (si-NC) were synthesized by GenePharma (Shanghai, China). For overexpression of integrin 35 (ITGB5), full-length cDNA of ITGB5 was with pc DNA3.1 vector, and the empty vector was used as the control. miR-221-5p mimics, negative control (NC) mimics, miR-221-5p inhibitor and NC inhibitor were obtained from GenePharma.. Cell transfections were conducted by using Lipofectamine 2000 Reagent (Life Technologies Corporation, Carlsbad) in accordance with the manufacturers' instruction.

\section{Quantitative real-time PCR}

Total RNAs were extracted using TRIzol Reagent (Invitrogen, Thermo

Fisher Scientific, Inc., Waltham, MA) from tissues or cells. Complementary DNAs were generated by using RNAs as templates through the Revert Aid First Strand cDNA Synthesis Kit (Thermo Fisher Scientific, Waltham, MA). qPCR was analyzed using the SYBR Green PCR Master Mix Kit (Takara Biotechnology Co., Ltd., Dalian, China) on an ABI 7500 Real-Time PCR System (Applied Biosystems). The relative expression was normalized to glyceraldehyde 3-phosphate dehydrogenase (GAPDH) or U6 and calculated according to the $2^{-{ }^{\triangle}} \mathrm{Ct}$ method. The primer sequences were listed as follows: RNF185-AS1 (forward: $5^{\prime}-$ AAGCCACATGTCTCACCAGG-3' and reverse: 5'-GCCGCTCTGTTCTCTTTCCT-3'). miR-221-5p (forward: 5'- 
ACACTCCAGCTGGGACCTGGCATACAATGT-3' and reverse: 5'-CTCAACTGGTGTCGTGGA-3'); GAPDH (forward: 5'-GCTCCCTCTTTCTTTGCAGC-3' and reverse: 5'-GGAAAGCCAGTCCCCAGAAC-3'); U6 forward:

5'- CTCGCTTCGGCAGCACA-3' and reverse: 5'-AACGCTTCACGAATTTGCGT-3').

\section{Subcellular fractionation assay}

The cytoplasmic and nuclear extracts were extracted from HCC cells with NE-PER Nuclear and Cytoplasmic Extraction Reagents (Thermo Fisher Scientific, Waltham, MA). The distribution of RNF185AS1 in cytoplasm or nucleus was analyzed by qRT-PCR analysis. The expression of U6 in nucleus, GAPDH in cytoplasm was used as control.

\section{Cell counting kit-8(CCK-8)}

The cell proliferation was determined using CCK-8 kit (DoJinDo, Shiga, Japan). HepG2 and Huh-7 cells $\left(2.5 \times 10^{3}\right.$ cells per well) were plated into 96 well plates after $48 \mathrm{~h}$ transfection. After incubation for 0,24 , 48 and 72 hours, 10 ul of CCK-8 solution was added and determined at a wavelength of $450 \mathrm{~nm}$.

\section{Colony formation assay}

HepG 2 and Huh-7 cells $\left(1 \times 10^{3}\right.$ cells per well) were plated into 6 well plates after $48 \mathrm{~h}$ transfection and cultured with fresh growth medium every 3 days. Cell were cultured for 21 days and were stained with $0.5 \%$ crystal violet.

\section{Cell migration and invasion assays}

For analysis of cell migration and invasion, $2.0 \times 10^{4}$ cells were seeded in a transwell chamber (8- $\mu \mathrm{m}$ pore size; EMD Millipore, Billerica, MA, USA) with $200 \mu \mathrm{L}$ serum-free DMEM. A volume of $500 \mu \mathrm{L}$ of the medium containing $10 \%$ FBS was added to the lower chamber. The 24 -well chambers were then incubated at $37^{\circ} \mathrm{C}$ for $24 \mathrm{~h}$, the cells on the lower surface of the membrane were fixed in $4 \%$ paraformaldehyde for 30 minutes and stained with $0.5 \%$ crystal violet. The transmembrane cells were counted under six random microscope fields.

\section{Western blot analysis}

Total proteins were prepared using RIPA lysis buffer (Beyotime, China). Proteins were quantified by BCA assay. Equal amounts of protein were separated by $10 \%$ SDS-PAGE and electrotransferred onto polyvinylidene difluoride membranes (Bio-Rad, USA). After being blocked using $5 \%$ non-fat milk for $1 \mathrm{~h}$ at room temperature, the primary rabbit anti-human antibodies against E-cadherin (ab1416, Abcam, Cambridge, MA), Vimentin (ab92547, Abcam), integrin $\beta 5$ (ab184312, Abcam) and GAPDH (ab9485, Abcam) were supplemented overnight at $4^{\circ} \mathrm{C}$. Then the members were washed with tris-buffered saline Tween 20 for three times and probed with horse radish peroxidase-conjugated secondary antibodies 
(ab205718, Abcam) for $1 \mathrm{~h}$ at $37^{\circ} \mathrm{C}$. The bands were visualized using a ChemicDocXRS system (Bio-Rad, USA).

\section{Luciferase reporter assay}

For luciferase reporter assay, the predicted sequences of RNF185-AS1 or integrin $\beta 5$ were inserted into pGL3 vector (Promega, Madison, WI, USA). Afterward, these vectors were co-transfected with miR-221-5p mimics and its corresponding control into Huh7 and HepG2 cells. 48 hours later, luciferase reporter assay system (Promega) was performed to measure the luciferase activities.

\section{RNA-binding protein immunoprecipitation (RIP) assay}

RIP was carried out by using the EZMagna RIP kit (Millipore, Bedford, MA, USA) according to the manufacturer's instructions. In brief, cells were lysed and the lysates were incubated with magnetic beads conjugated with human anti-Ago2 antibody (ab57113, Abcam) or control anti-IgG (ab131368, Abcam). Immunoprecipitated RNAs were isolated by proteinase $\mathrm{K}$ and the expression of precipitated miRNAs was analyzed by qRT-PCR.

\section{Xenograft mouse model}

Nude mice (female BALB/c-nu, 6 weeks old) were obtained from the National Laboratory Animal Center (Beijing, China). All experimental and animal care procedures were approved by the Animal Research Ethics Committee of Wuhan No.1 Hospital. The subcutaneous xenograft model was established by directly subcutaneously injecting $2 \times 10^{6}$ Huh7 cells stably transfected with si-NC or si-RNF185-AS1. Ki-67, ITGB5, E-cadherin and Vimentin immunohistochemistry analysis were conducted.

For the tumor metastasis model, $1 \times 10^{6}$ Huh7 cells transfected with si-NC or si-RNF185-AS1 were injected into mouse tail veins ( $n=6$ for each group). The mice were killed at the indicated time. Lungs were surgically retrieved from mice. Micrometastatic tumors in the lungs were counted. Tissues were embedded in paraffin, sectioned, stained with H\&E.

\section{Immunohistochemistry (IHC)}

Tissue sections were prepared and subjected to immunohistochemical analysis. Anti-human Ki67 (ab15580هAbcam), ITGB5 (ab15459, Abcam), E-cadherin and Vimentin antibody was used as primary antibodies. HRP-conjugated secondary Ab was used as secondary antibody. Images were obtained using an Olympus-BX51 microscope.

\section{Statistical analysis}

All data of multiple experiments are presented as the mean \pm standard deviation. SPSS 21.0 software (SPSS, Chicago, IL) was adopted for statistical analysis. The Kaplan-Meier analysis and log-rank test 
were used to calculate the overall survival. The one-way ANOVA or student's t test was adopted for the differences among groups. The value of $p<0.05$ was considered to be significant.

\section{Results}

\section{RNF185-AS1 is upregulated in in HCC}

GEPIA database was utilized to select the upregulated IncRNA in HCC, higher expression of RNF185-AS1 was found in HCC tissues than that in normal nontumor tissues (Fig. 1a,b). The expression of RNF185AS1 in HCC patients serum and HCC cell lines was measured by qRT-PCR. We found that RNF185-AS1 expression was increased in the serum of HCC patients compared with health controls (Fig. 1C). qRT-PCR results showed that RNF185-AS1 was up-regulated in HCC cell lines when compared with human normal liver cell line HL7702 (Fig. 1d). Kaplan-Meier analysis showed that patients with high levels of RNF185AS1 had a lower survival rate than those with low levels (Fig. 1e). Further analysis indicated that high levels of RNF185-AS1 were positively correlated with advanced TNM stage, distant metastasis (Fig. 1f, g), suggesting that RNF185-AS1 may promote HCC progression and metastasis.

\section{RNF185-AS1 promotes HCC cell proliferation, migration, invasion and EMT}

To further explored the biological role of RNF185-AS1 in HCC cells. RNF185-AS1 was successfully silenced in Huh7 and HepG2 cells by transfection with si-RNF185-AS1 (Fig. 2a). CCK-8 assay showed that the proliferative ability of cells was remarkably declined by downregulation of RNF185-AS1 in HCC cells (Fig. 2b). Colony formation assay revealed that RNF185-AS1 knockdown inhibited cell proliferation (Fig. 2c). Matrigel assay showed that si-RNF185-AS1 significantly inhibited Huh7 and HepG2 cells migration and invasion (Fig. 2d, e). Furthermore, knockdown of RNF185-AS1 increased E-cadherin and inhibited Vimentin expression in Huh7 and HepG2 cells (Fig. 2f). These results suggested that RNF185-AS1 promotes HCC cells proliferation, migration, invasion and EMT.

\section{RNF185-AS1 is a sponge for miR-221-5p}

We next investigate the regulatory mechanism of RNF185-AS1. Subcellular fractionation assay showed that RNF185-AS1 mainly localized in the cytoplasm of HCC cells (Fig. 3a). According to the prediction from Starbase, we found that miR-221-5p had binding sites for RNF185-AS1 (Fig. 3b). We then performed luciferase reporter assay to confirm the correlation between RNF185-AS1 and miR-221-5p. The results showed that miR-221-5p mimics reduced the luciferase activity of RNF185-AS1-Wt, but did not affect that of mutant RNF185-AS1-Mut (Fig. 3c). RIP assay showed that RNF185-AS1 and miR-221-5p were notably enriched in Ago2 groups compared to control IgG groups (Fig. 3d). Moreover, Pearson correlation analysis showed that miR-221-5p expression negatively related with RNF185-AS1 expression in HCC tissues (Fig. 3e). miR-221-5p was significantly upregulated in cells transfected with si-RNF185-AS1 (Fig. 3f), indicating the negative regulation of RNF185-AS1 on miR-221-5p. Thus, we demonstrated that RNF185-AS1 was a sponge for miR-221-5p in HCC. 


\section{Integrin $\beta 5$ acts as a target gene of miR-221-5p in HCC}

We used TargetScan (www.targetscan.org) database to predicate the potential targets of miR-221-5p and identified integrin $\beta 5$ (ITGB5) was a potential target of miR-221-5p (Fig. 4a). Luciferase activity assay showed that miR-221-5p mimics decreased the luciferase activity of ITGB5-Wt, while no change was observed in the luciferase activity of ITGB5-Mut (Fig. 4b). Additionally, the RIP assay suggested that miR221-5p and ITGB5 were enriched in Ago2 groups as compared with the IgG groups (Fig. 4c), suggesting ITGB5 was a target gene of miR-221-5p. Furthermore, we found that ITGB5 expression was inhibited by miR-221-5p mimics (Fig. 4d). To further determine whether RNF185-AS1 regulates ITGB5 expression through inhibiting miR-221-5p expression, we transfected HCC cell with si-RNF185-AS1, si-RNF185-AS1+ miR-221-5p inhibitor. The results showed that si-RNF185-AS1 inhibited ITGB5 expression (Fig. 4e), whereas miR-221-5p inhibitor restored the expression of ITGB5 inhibited by si-RNF185-AS1 (Fig. 4f). We found that ITGB5 was overexpressed in HCC tissues (Fig. 4g). Pearson's correlation analysis showed that there was an inverse correlation between miR-221-5p and ITGB5, and RNF185-AS1 was positively related to ITGB5 in HCC tissues (Fig. 4h, i). Taken together, these results suggested that RNF185-AS1 promote ITGB5 expression by regulating miR-221-5p in HCC.

\section{RNF185-AS1 promoted HCC progression through regulating miR-221-5p/ITGB5}

To investigate whether RNF185-AS1 promoted HCC cells proliferation, migration, invasion and EMT through miR-221-5p/ITGB5 axis, we transfected Huh7 cells with si-RNF185-AS1, miR-221-5p inhibitor + si-RNF185-AS1 or si-NC+ control inhibitor. Then CCK8 assay, colony formation assay, transwell assays and western blot were performed. We found that RNF185-AS1 knockdown inhibited proliferation, migration, invasion and EMT of Huh7 cells, whereas miR-221-5p inhibitor reversed the effects of siRNF185-AS1 (Fig. 5a-d). Furthermore, we restored the expression of ITGB5. Results showed that ITGB5 restoration rescued the abilities of proliferation, migration, invasion and EMT in RNF185-AS1-depleted Huh7 cells. (Fig. 5e-h). Taken together, these results demonstrated that RNF185-AS1 regulates HCC progression through miR-221-5p/ITGB5 pathway.

\section{RNF185-AS1 knockdown inhibits HCC progression in vivo}

Finally, we investigated the roles of RNF185-AS1 in vivo by xenograft experiments. We found that tumors from the si-RNF185-AS1 group showed smaller tumor weight than those from the control group (Fig. 6aC). IHC assay showed that Ki67 and ITGB5 expression was significantly decreased in the group of siRNF185-AS1 compared with controls (Fig. 6d). The expression of E-cadherin increased while the expression of vimentin decreased obviously in the si-RNF185-AS1 group (Fig. 6d). The miR-221-5p level in xenograft tumors was significantly increased in the si-RNF185-AS1 group (Fig. 6e). In lung metastasis models, the mice injected with Huh7 cells which transfected with si-RNF185-AS1 exhibited less metastatic nodules in the lungs than those injected with the control cells (Fig. 6f). These results suggested that RNF185-AS1 promote HCC cell growth and metastasis in vivo. 


\section{Discussion}

Mounting evidences have shown that abnormal expression of IncRNAs is involved in the progression of HCC [9-11]. In the present study, we identified the oncogenic roles of RNF185-AS1 in HCC. We found that RNF185-AS1 expression was overexpressed in HCC and positively correlated with TNM stage, metastasis and low survival rate. We demonstrated that RNF185-AS1 knockdown suppressed the HCC cell proliferation, migration, invasion, EMT in vitro. Furthermore, we found that RNF185-AS1 knockdown inhibited HCC cell growth and metastasis in vivo. We further identified that RNF185-AS1 acts as a ceRNA for miR-221-5p and upregulated integrin $\beta 5$ expression.

Many studies have reported that IncRNAs can interact with miRNA to exert their regulatory functions $[12,13]$. For example, LINC01296 promotes HCC cells proliferation, migration, and invasion through targeting miR-122-5p [14]. Long noncoding RNA SNHG1 promotes non-small cell lung cancer progression via sponging miR-145-5p [15]. Long noncoding RNA HAGLROS promotes HCC cells proliferation through regulating miR-5095/ATG12 axis [16]. In the present study, we assumed RNF185-AS1 to act as a ceRNA, by using bioinformatics tools, we found that sequence RNF185-AS1 contained the binding sites for miR221-5p. Luciferase reporter assay and RIP assay verified the prediction that RNF185-AS1 directly interacted with miR-221-5p. miR-221-5p has been reported to exerts tumor suppressive function in prostate cancer [17,18], gastric cancer [19] and colorectal cancer [20]. On the contrary, previous research have reported that miR-221-5p acted as an oncogene in several tumors, such as renal cell cancer [21], prostate cancer [22]. However, the role of miR-221-5p in HCC has not been studied. In our study, we found that miR-221-5p expression was negatively regulated by RNF185-AS1. miR-221-5p inhibitor reversed RNF185-AS1-induced biological effects. Therefore, we concluded that RNF185-AS1 is a sponge for miR221-5p in HCC.

IGTB5 is a member of integrin family and has been reported to regulate diverse biological functions in cancers, including proliferation, EMT, migration, invasion and angiogenesis. It has been reported that ITGB5 cooperated with endothelial growth factor receptor 2(VEGF2) to enhance cell survival [23]. Previous studies have shown that integrin $\beta 5$ ITGB5 promotes cancer cell migration, invasion and contributes to the transforming growth factor $\beta$ (TGF- $\beta$ )-induced EMT [24-25]. Studies have revealed that ITGB5 facilitates tumor angiogenesis [26, 27]. A recent study reported that miR-185-ITGB5- $\beta$-catenin pathway plays an important role in HCC tumorigenesis [28]. In our study, we found that the expression of ITGB5 was increased in HCC. We demonstrated the direct interaction between ITGB5 and miR-221-5p in HCC. We also revealed that ITGB5 expression was inhibited by miR-221-5p and upregulated by RNF185AS1. Restoration of ITGB5 significantly rescued the proliferation, migration, invasion and EMT of HCC cells transfected with si-RNF185-AS1.

\section{Conclusion}

Our study provided evidence that RNF185-AS1 promotes HCC progression through miR-221-5p/ITGB5. Our results indicated a novel mechanism of RNF185-AS1 in developments of HCC, which plays a critical 
role in HCC progression and may be an oncogene in HCC cells.

\section{Declarations}

\section{Acknowledgements}

None.

\section{Funding}

This work was supported by the following funds: National Natural Science Foundation of China (No. 81502222)

\section{Author contributions}

JH Zhu and JY Yang conceived and designed the experiments; CM Huang, K Li and RF Huang performed the experiments; RF Huang and CM Huang analyzed the data; CM Huang wrote the paper. All authors contributed to data analysis, drafting or revising the article, gave final approval of the version to be published, and agree to be accountable for all aspects of the work.

\section{Availability of data and materials}

The datasets used during this research are available from the corresponding author upon reasonable request.

\section{Ethics approval and consent to participate}

The present study was approved by the Ethics Committee of Wuhan No.1 Hospital. The research has been carried out in accordance with the World Medical Association Declaration of Helsinki. All patients provided written informed consent.

\section{Consent for publication}

Not applicable.

\section{Competing interests}

The authors declare that they have no competing interests.

\section{References}

1. J. Bruix, G.J. Gores, V. Mazzaferro. Hepatocellular carcinoma: clinical frontiers and perspectives. Gut. 2014; 63: 844-55.

2. Spizzo R, Almeida MI, Colombatti, Calin GA. Long non-coding RNAs and cancer: A new frontier of translational research? Oncogene. 2012; 31: 4577-87. 
3. Chen J, Liu S, Hu X. Long non-coding RNAs: crucial regulators of gastrointestinal cancer cell proliferation. Cell Death Discov. 2018; 4 : 1.

4. Xiao H, Zhang F, Zou Y, Li J, Liu Y, Huang W. The function and mechanism of long non-coding RNAATB in cancers. Front Physiol. 2018; 9: 321.

5. Lian Y, Xiong F, Yang L, Bo H, Gong Z, Wang Y, et al. Long noncoding RNA AFAP1-AS1 acts as a competing endogenous RNA of miR-423-5p to facilitate nasopharyngeal carcinoma metastasis through regulating the Rho/Rac pathway. J Exp Clin Cancer Res. 2018; 37(1): 253.

6. Ji D, Wang Y, Sun B, Yang J, Luo X. Long non-coding RNA MNX1-AS1 promotes hepatocellular carcinoma proliferation and invasion through targeting miR-218-5p/COMMD8 axis. Biochem Biophys Res Commun. 2019; 3 669-674.

7. Li Y, Zhuang W, Huang M, Li X. Long noncoding RNA DDX11-AS1 epigenetically represses LATS2 by interacting with EZH2 and DNMT1 in hepatocellular carcinoma. Biochem Biophys Res Commun. 2019; 4: 1051-1057.

8. Chen K, Zhang L. LINC00339 regulates ROCK1 by miR-152 to promote cell proliferation and migration in hepatocellular carcinoma. J Cell Biochem. 2019; 9: 14431-14443.

9. Lin Y, Jian Z, Jin H, Wei X, Zou X, Guan R, et al. Long non-coding RNA DLGAP1-AS1 facilitates tumorigenesis and epithelial-mesenchymal transition in hepatocellular carcinoma via the feedback loop of miR-26a/b-5p/IL-6/JAK2/STAT3 and Wnt/ $\beta$-catenin pathway. Cell Death and Disease. 2020; 11: 34 .

10. Zhang L, Niu H, Ma J, Yuan B, Chen Y, Zhuang Y, et al. The molecular mechanism of LncRNA34amediated regulation of bone metastasis in hepatocellular carcinoma. Mol. Cancer. 2019; 18:120.

11. Kong Q, Liang C, Jin Y. The IncRNA MIR4435-2HG is upregulated in hepatocellular carcinoma and promotes cancer cell proliferation by upregulating miRNA-487a. Cell Mol Biol Lett. 2019; 24: 26.

12. Wang Y, Liu Z, Yao B, Li Q, Wang L, Wang C, et al. Long non-coding RNA CASC2 suppresses epithelialmesenchymal transition of hepatocellular carcinoma cells through CASC2/miR-367/FBXW7 axis. Mol. Cancer. 2017; 16: 123.

13. Yamamura S, Imai-Sumida M, Tanaka Y, Dahiya R. Interaction and cross-talk between non-coding RNAs. Cell Mol Life Sci. 2018; 75(3): 467-84.

14. Wan Y, Li M, Huang P. LINC01296 promotes proliferation, migration, and invasion of HCC cells by targeting miR-122-5P and modulating EMT activity. Onco Targets Ther. 2019; 12: 2193-2203.

15. Lu Q, Shan S, Li Y, Zhu D, Jin W, Ren T. Long noncoding RNA SNHG1 promotes non-small cell lung cancer progression by up-regulating MTDH via sponging miR-145-5p. FASEB J. 2018; 32(7): 3957-67.

16. Wei H, Hu J, Pu J, Tang Q, Li W, Ma R. Long noncoding RNA HAGLROS promotes cell proliferation, inhibits apoptosis and enhances autophagy via regulating miR-5095/ATG12 axis in hepatocellular carcinoma cells. International Immunopharmacology. 2019; 73: 72-80.

17. Kiener M, Chen L, Krebs M, Grosjean J, Klima I, Kalogirou C, et al. miR-221-5p regulates proliferation and migration in human prostate cancer cells and reduces tumor growth in vivo. BMC Cancer. 2019; 
19: 627.

18. Kneitz B, Krebs M, Kalogirou C, Schubert M, Joniau S, van Poppel H, et al. Survival in patients with high-risk prostate cancer is predicted by miR-221, which regulates proliferation, apoptosis, and invasion of prostate cancer cells by inhibiting IRF2 and SOCS3. Cancer Res. 2014; 74(9): $2591-603$.

19. Jiang X, Jiang M, Guo S, Cai P, Wang W, Li Y. Promotion of miR-221-5p on the Sensitivity of Gastric Cancer Cells to Cisplatin and Its Effects on Cell Proliferation and Apoptosis by Regulating DDR1. Onco Targets Ther. 2020; 13: 2333-45.

20. Yuan K, Xie K, Fox J, Zeng H, Gao H, Huang C, et al. Decreased levels of miR-224 and the passenger strand of miR-221 increase MBD2, suppressing maspin and promoting colorectal tumor growth and metastasis in mice. Gastroenterology. 2013; 145(4): 853-64.

21. Liu S, Wang Y, Li W, Yu S, Wen Z, Chen Z, et al. miR-221-5p acts as an oncogene and predicts worse survival in patients of renal cell cancer. Biomed Pharmacother. 2019; 119: 109406.

22. Shao N, Ma G, Zhang J, Zhu W. miR-221-5p enhances cell proliferation and metastasis through posttranscriptional regulation of SOCS1 in human prostate cancer. BMC Urol. 2018; 18(1): 14.

23. Tancioni I, Uryu S, Sulzmaier FJ, Shah NR, Lawson C, Miller NL, et al. FAK inhibition disrupts a beta5 integrin signaling axis controlling anchorage-independent ovarian carcinoma growth. Mol Cancer Ther. 2014; 13: 2050-61.

24. Bianchi A, Gervasi ME, Bakin A. Role of beta5-integrin in epithelial

-esenchymal transition in response to TGF-beta. Cell Cycle. 2010; 9: 1647-59.

25.Majhen D, Stojanovic N, Speljko T, Brozovic A, De Zan T, Osmak M, et al. Increased expression of the coxsackie and adenovirus receptor downregulates alphavbeta3 and alphavbeta5 integrin expression and reduces cell adhesion and migration. Life Sci. 2011; 89: 241-9.

26. Bianchi-Smiraglia A, Paesante S, Bakin AV. Integrin beta 5 contributes to the tumorigenic potential of breast cancer cells through the Src-FAK and MEKERK signaling pathways. Oncogene. 2013; 32: 304958.

27. Li F, Liu Y, Kan X, Li Y, Liu M, Lu JG. Elevated expression of integrin alphav and beta5 subunit in laryngeal squamous-cell carcinoma associated with lymphatic metastasis and angiogenesis. Pathol Res Pract. 2013; 209: 105-9.

28. Lin Z, He R, Luo H, Lu C, Ning Z, Wu Y, et al. Integrin- $\beta 5$, a miR-185-targeted gene, promotes hepatocellular carcinoma tumorigenesis by regulating $\beta$-catenin stability. J Exp Clin Cancer Res. 2018; 37: 17.

\section{Figures}


a

\begin{tabular}{|c|c|c|c|c|c|}
\hline Gene Symbol & Gene ID & Median (Tumor) & Median (Normal) & Log2(fold Change) & , adjp \\
\hline STESIA6-ASI & ENSG00000204832.9 & 3810 & 0.000 & 2266 & $287 \mathrm{e}-27$ \\
\hline BAFF185-AS1 & ENSG00000254835.1 & 3.090 & 0.212 & 1.755 & $6300-5$ \\
\hline ZFASI & ENSG00000177410.12 & 81.002 & 27.780 & 1.511 & $7.16 \mathrm{e}-40$ \\
\hline DLGAP1-AS1 & ENSG00000177337.7 & 7.940 & 2.765 & 1.248 & $3.17 e-30$ \\
\hline IRIM52-AS1 & ENSG00000248275.1 & 9.200 & 3.350 & 1.229 & $7070-50$ \\
\hline ELVCR1-AS1 & ENSG00000198468.7 & 2.930 & 0.710 & 1.201 & $1.73 e-32$ \\
\hline TOPORS-AS1 & ENSG00000235453.7 & 9.650 & 3.685 & 1.185 & $1.22 e-48$ \\
\hline SOX9.AS1 & ENSG00000234899.9 & 2.870 & 0.720 & 1,170 & $9.13 e-19$ \\
\hline
\end{tabular}

b

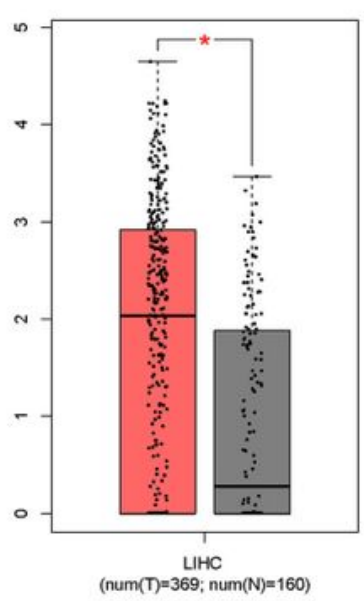

d

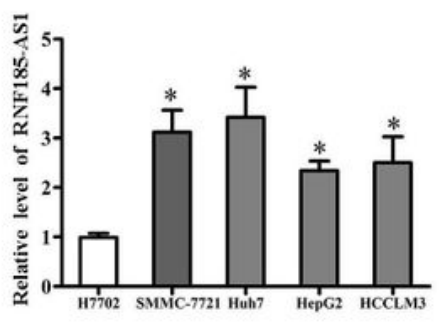

f

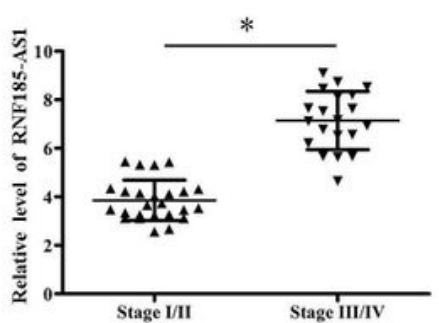

c

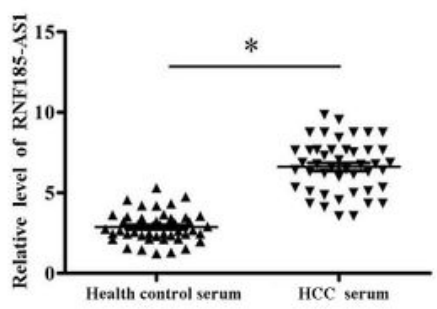

e

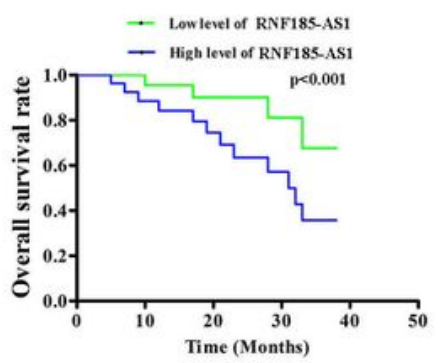

g

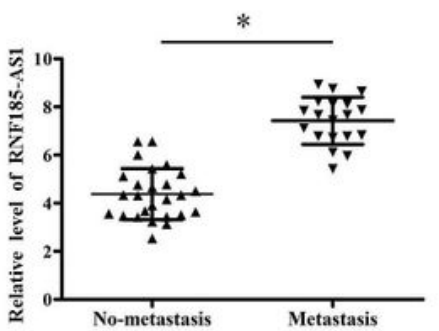

\section{Figure 1}

RNF185-AS1 was upregulated in HCC patients serum and HCC cell lines. a, b RNF185-AS1 upregulated in the HCC tissues was chosen from GEPIA database. c Increased expression of RNF185-AS1 in HCC tissues compared with normal tissues. d RNF185-AS1 was overexpressed in HCC cell lines. e Kaplan-Meier analysis was used to estimate the relation between RNF185-AS1 expression and overall survival of HCC 
patients. $\mathrm{f}, \mathrm{g}$ Upregulated expression of RNF185-AS1 was associated with advanced stage and distant metastasis. ${ }^{*} \mathrm{p}<0.05$

a

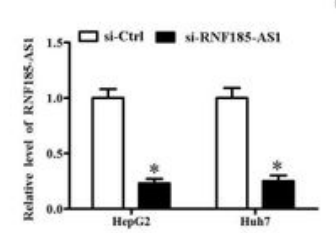

c
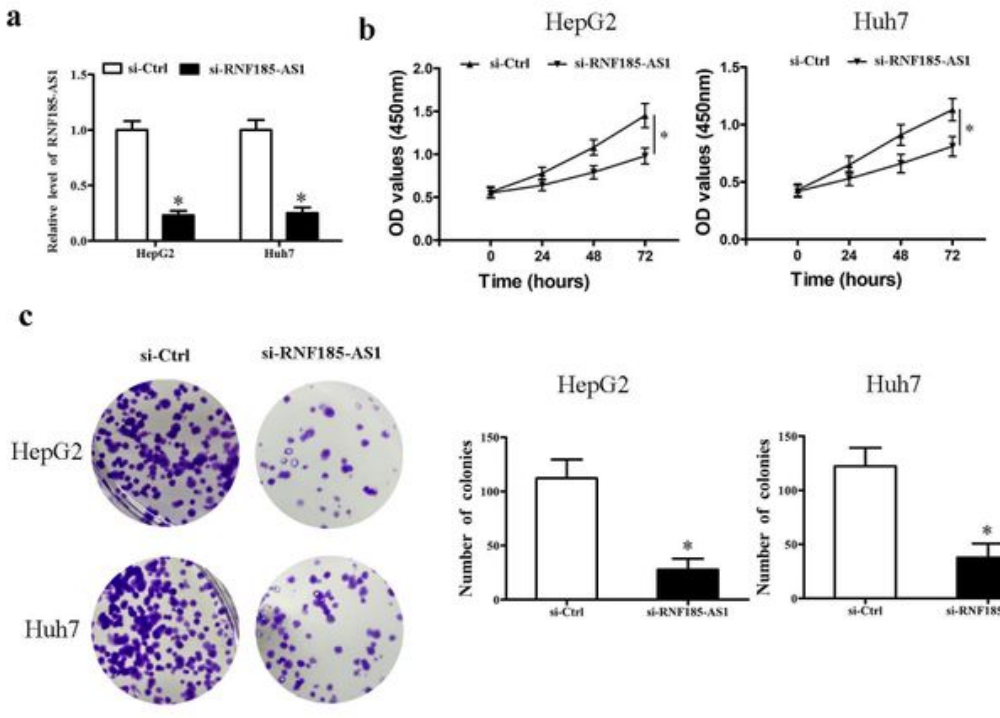

d
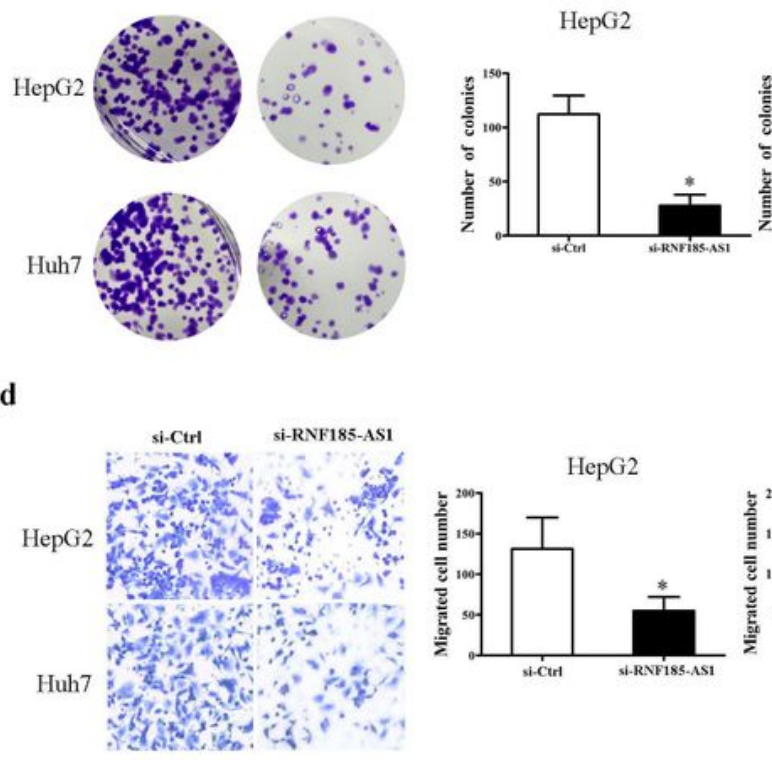

Huh7

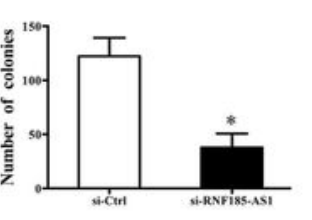

e
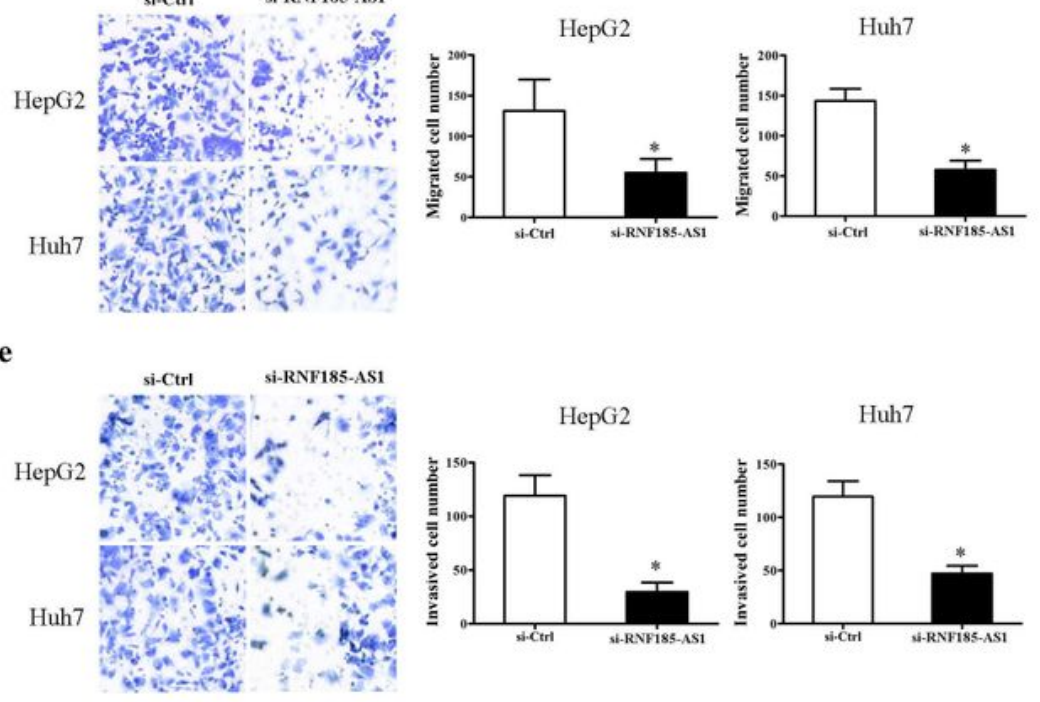

f

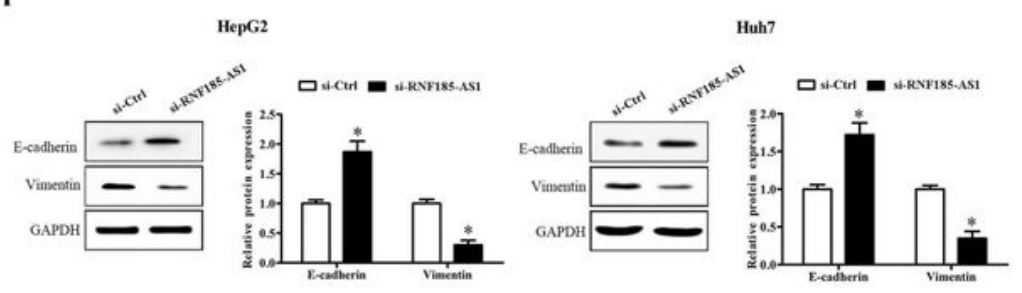

Figure 2

RNF185-AS1 promoted HCC cell proliferation, migration, invasion and EMT a RNF185-AS1 expression was decreased in Huh7 and HepG2 cells transfected with si-RNF185-AS1. b Cell proliferation was measured in Huh7 and HepG2 cells by CCK8 assay. c Colony formation assays were performed after 
RNF185-AS1 knockdown. d, e Transwell migration and invasion assay were performed after RNF185-AS1 knockdown. $\mathrm{f}$ Expressions of E-cadherin and Vimentin were detected by western blot in RNF185-AS1 knockdown Huh7 and HepG2 cells. * $p<0.05$.

a

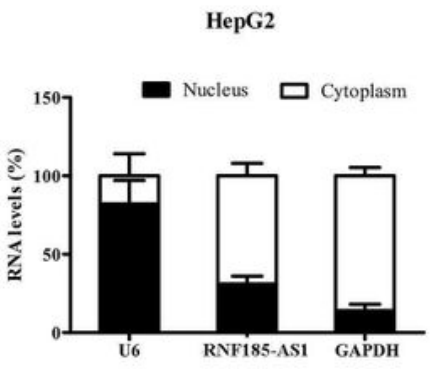

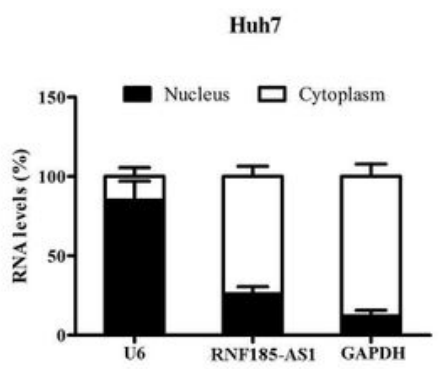

b

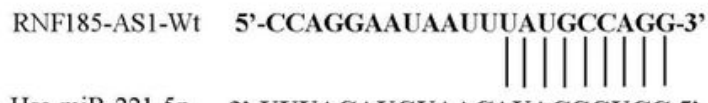

Hsa-miR-221-5p 3'-UUUAGAUGUAACAUACGGUCC-5'

RNF185-AS1-Mut 5'-CCAGGAAUAAUUCCCAAACCA-3'

c

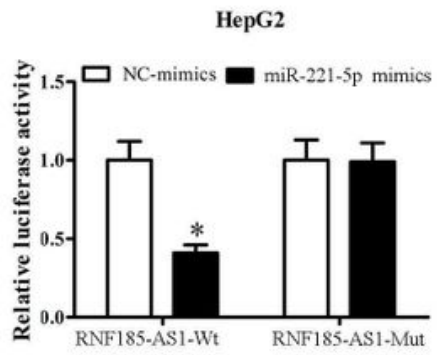

d

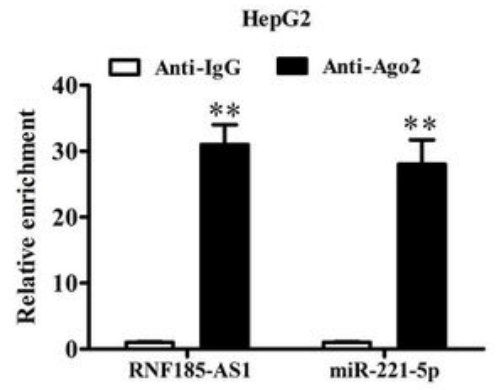

e

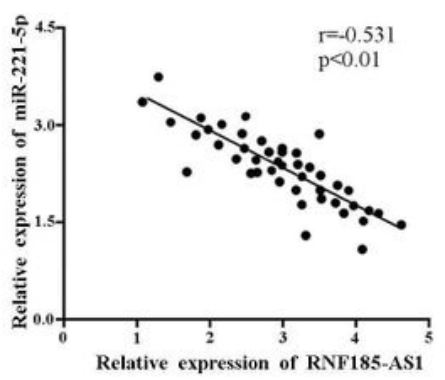

Huh7

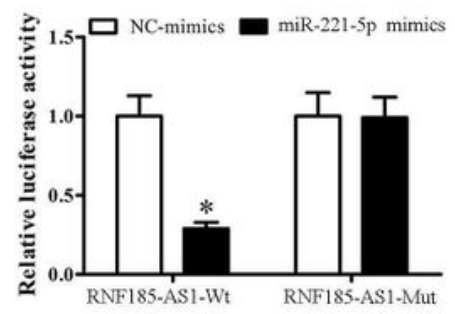

Huh7

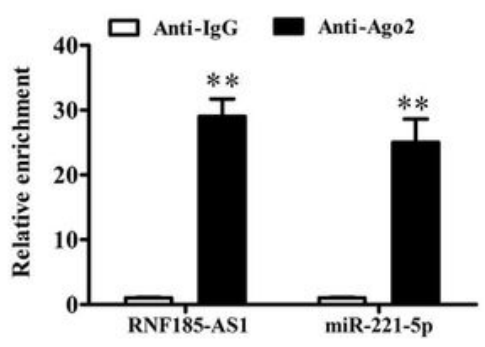

f

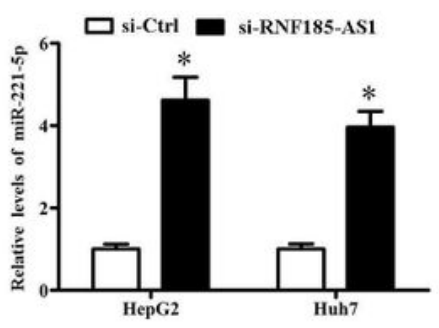

Figure 3

RNF185-AS1 was a sponge for miR-221-5p in HCC. a Subcellular fractionation assays was used to confirm that RNF185-AS1 located mainly in the cytoplasm. b The result of starBase predicted that miR- 
221-5p had a binding site for RNF185-AS1. c, d Luciferase reporter assay and RIP assay indicated that RNF185-AS1 could bind with miR-221-5p. e There was an inverse correlation between miR-221-5p and RNF185-AS1 in HCC tissues. f miR-221-5p expression was increased after RNF185-AS1 knockdown. * $p<0.05,{ }^{* *} p<0.01$.

a

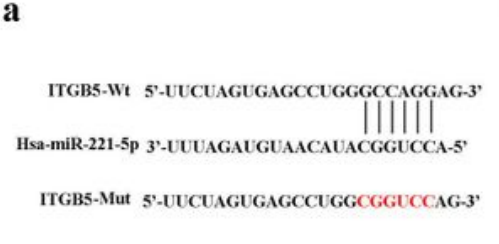

b

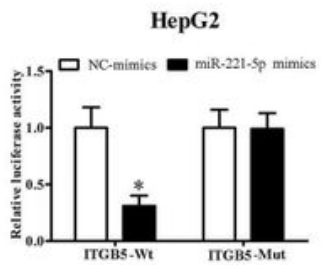

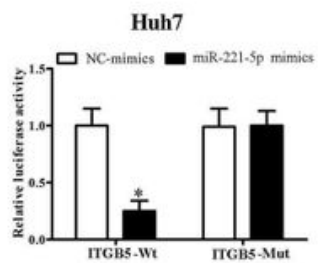

c
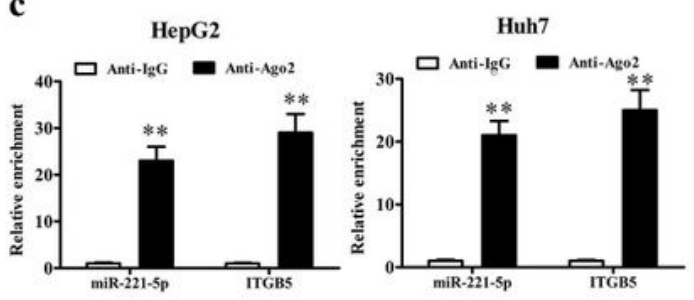

e
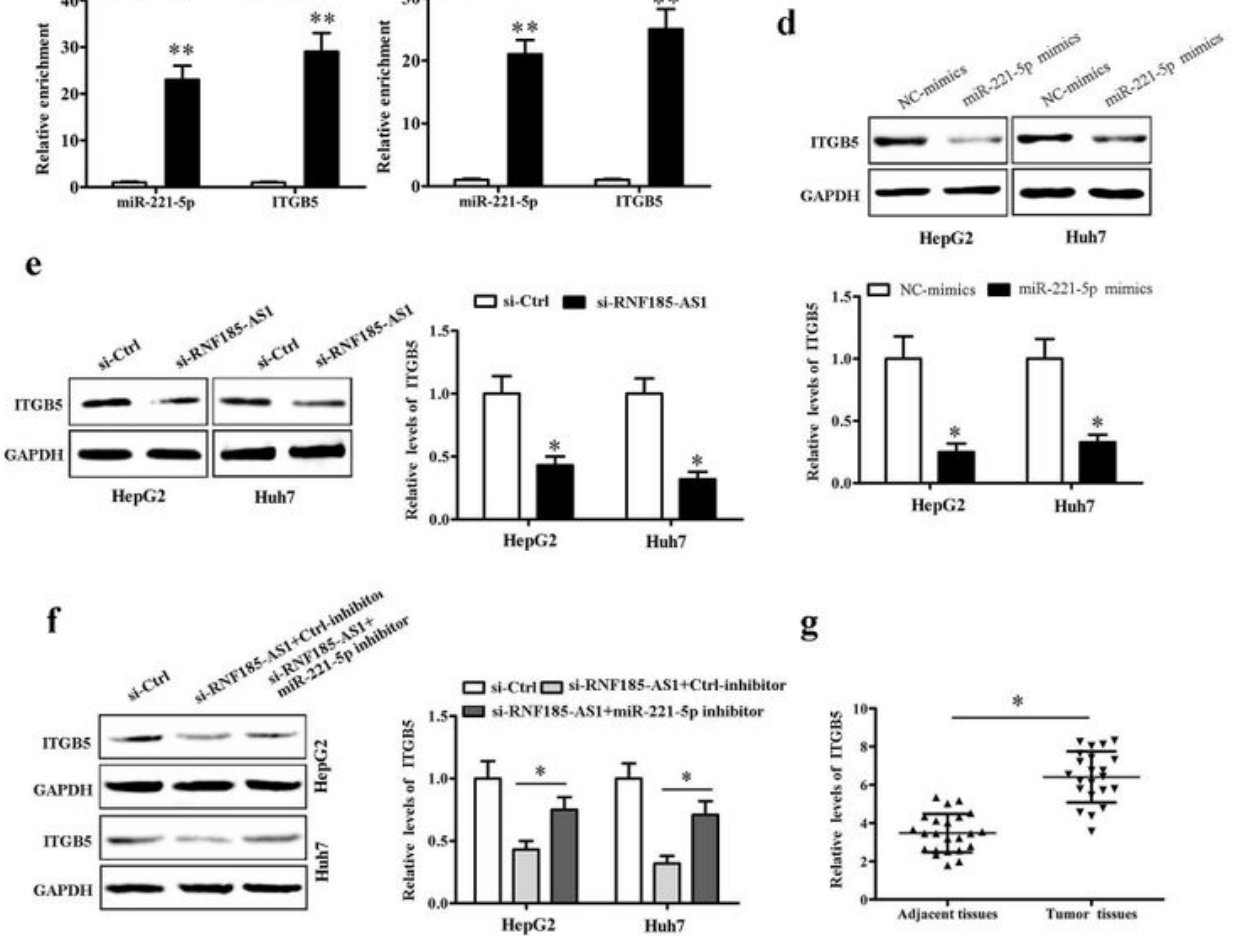

g

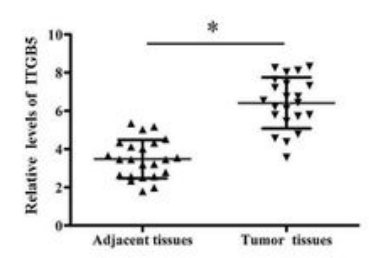

h

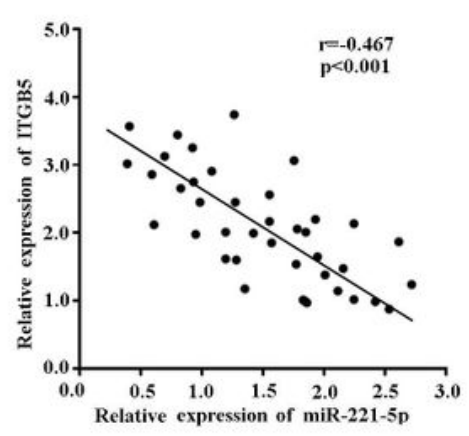

i

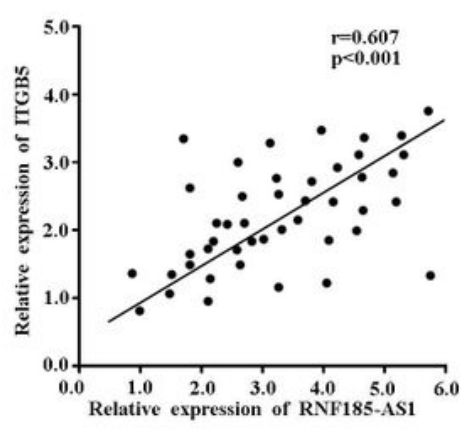

Figure 4 
miR-221-5p targeted ITGB5 in HCC. a Target Scan was used to predict that ITGB5 had a binding site for miR-221-5p. b Luciferase reporter assay verified that ITGB5 was a target gene of miR-221-5p. c The interaction between miR-221-5p and ITGB5 was confirmed by RIP assay. d, e ITGB5 expression was inhibited by miR-221-5p mimics or si-RNF185-AS1 in Huh7 and HepG2. f miR-221-5p inhibitor reversed the reduced-expression of ITGB5 caused by si-RNF185-AS1. g ITGB5 expression was upregulated in HCC tissues. h, i Pearson's correlation analysis was conducted to evaluate the correlation of ITGB5 and miR221-5p or RNF185-AS1 in HCC. * $p<0.05,{ }^{* *} p<0.01$.
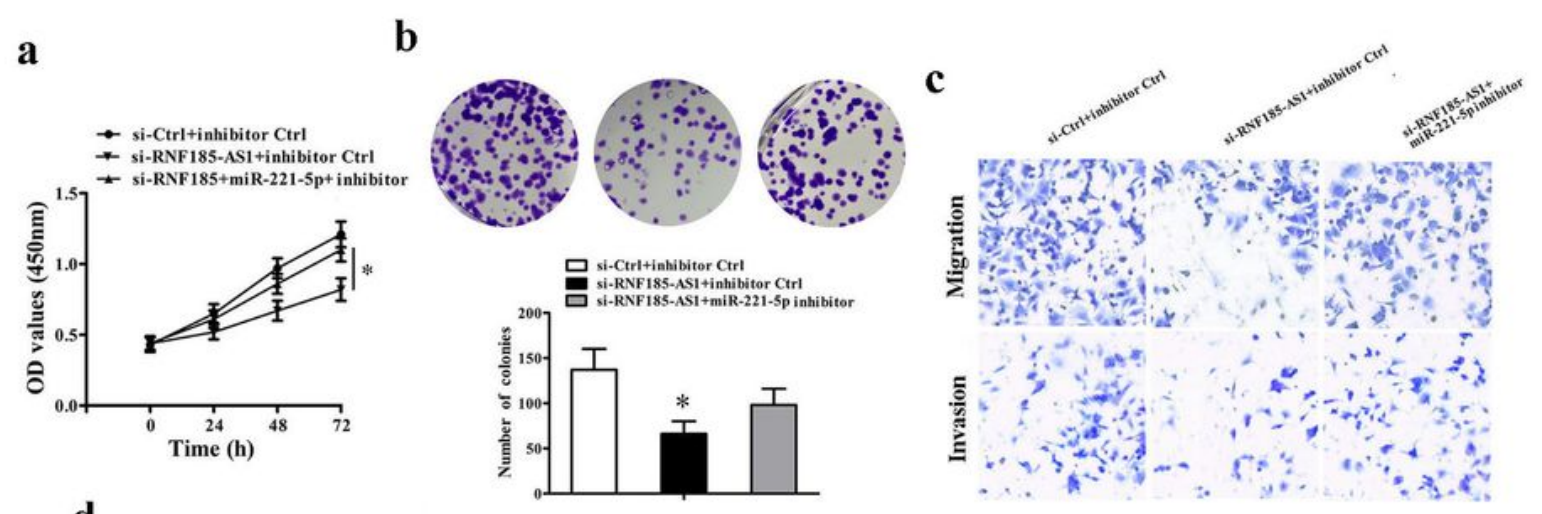

d
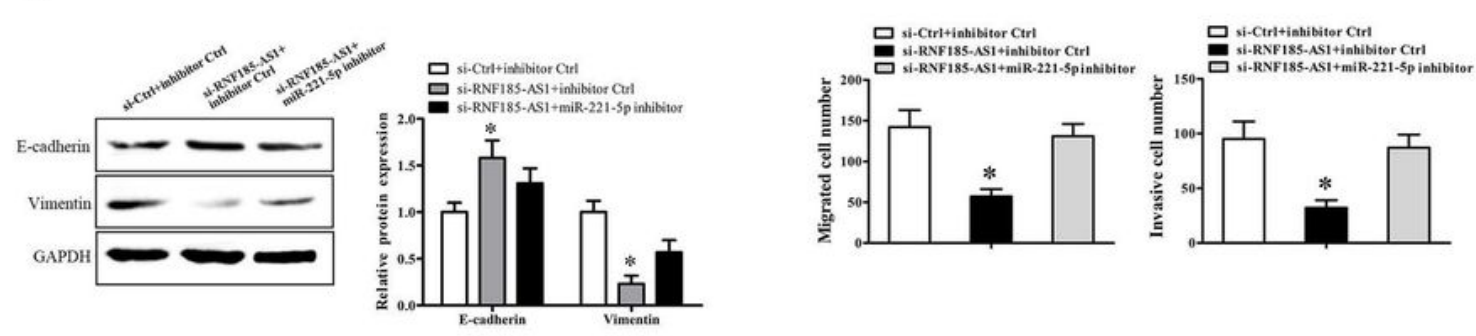

f
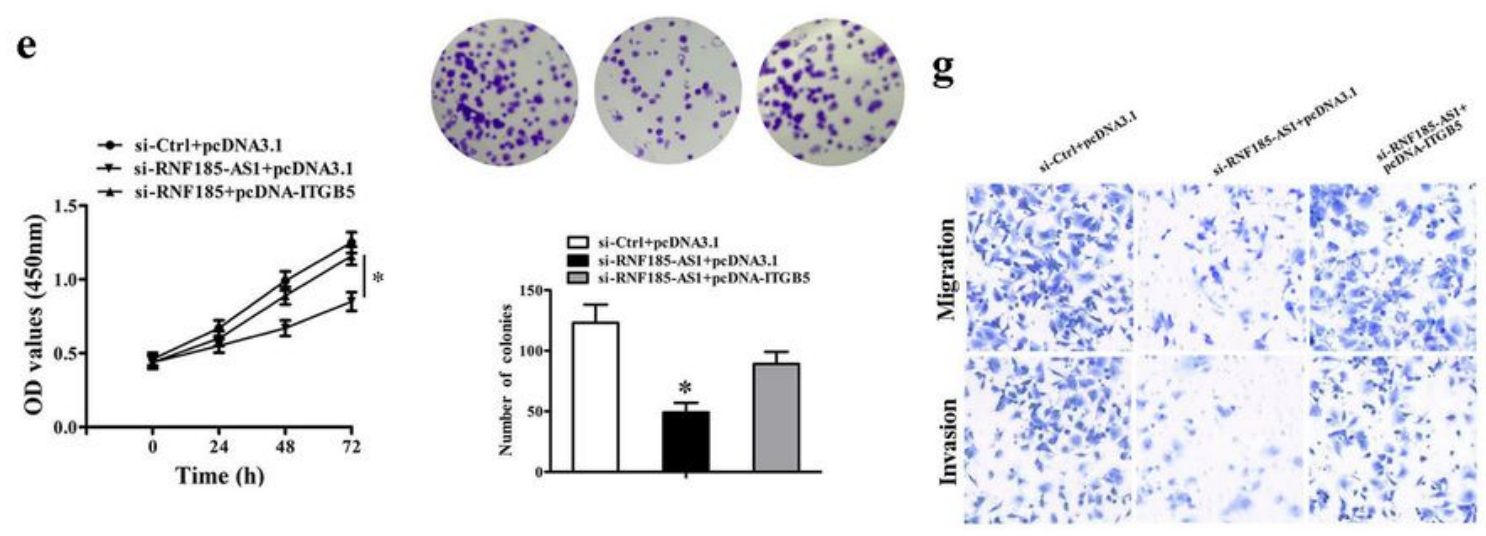

$\mathbf{h}$
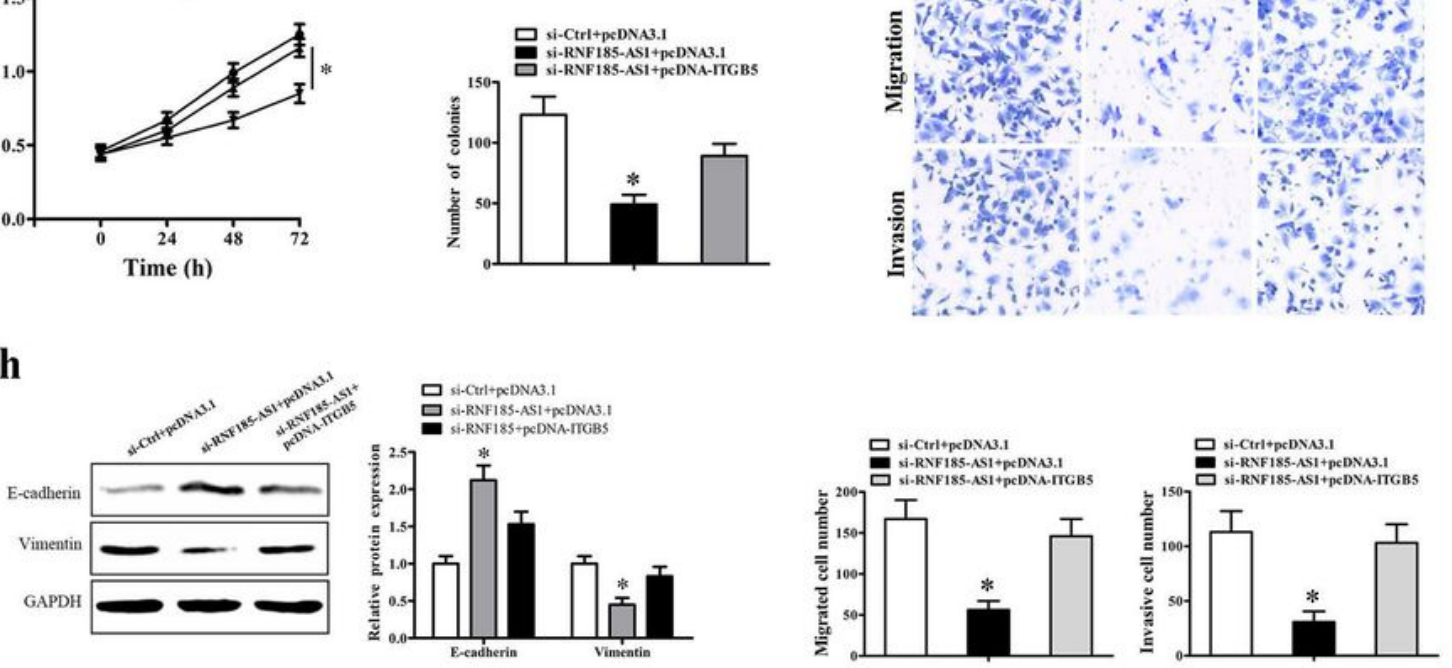


\section{Figure 5}

RNF185-AS1 promoted HCC progression through miR-221-5p/ITGB5 axis Huh7 cells were transfected with si-RNF185-AS1, miR-221-5p inhibitor + si-RNF185-AS1 or si-NC+control inhibitor. a, b CCK8 and colony formation assay were performed. c Migration and invasion assay was performed. $d$ EMT markers of cells were detected by western blot. Huh7 cells were transfected with si-RNF185-AS1, si-RNF185-AS1+ ITGB5-pcDNA3.1 or si-NC+pcDNA3.1. e, f CCK8 and colony formation assay were performed. g Migration and invasion assay was performed. $\mathrm{h}$ Expressions of E-cadherin and Vimentin were measured by western blot. 
a

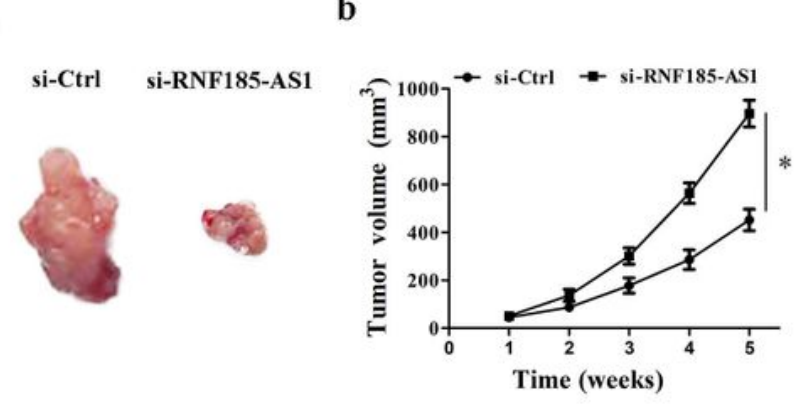

c

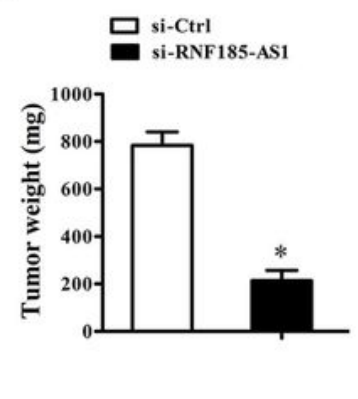

d
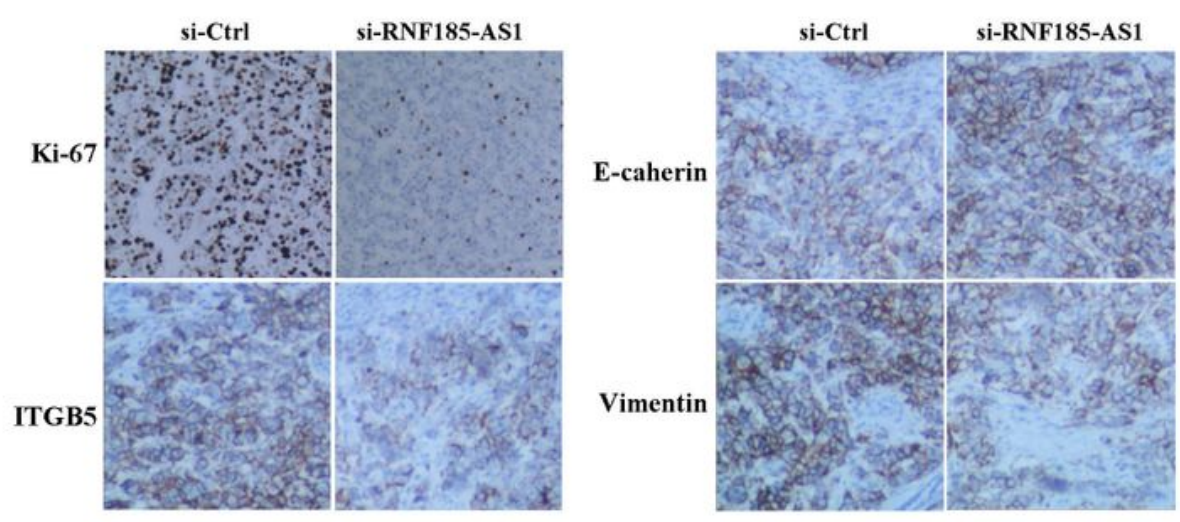

e

f
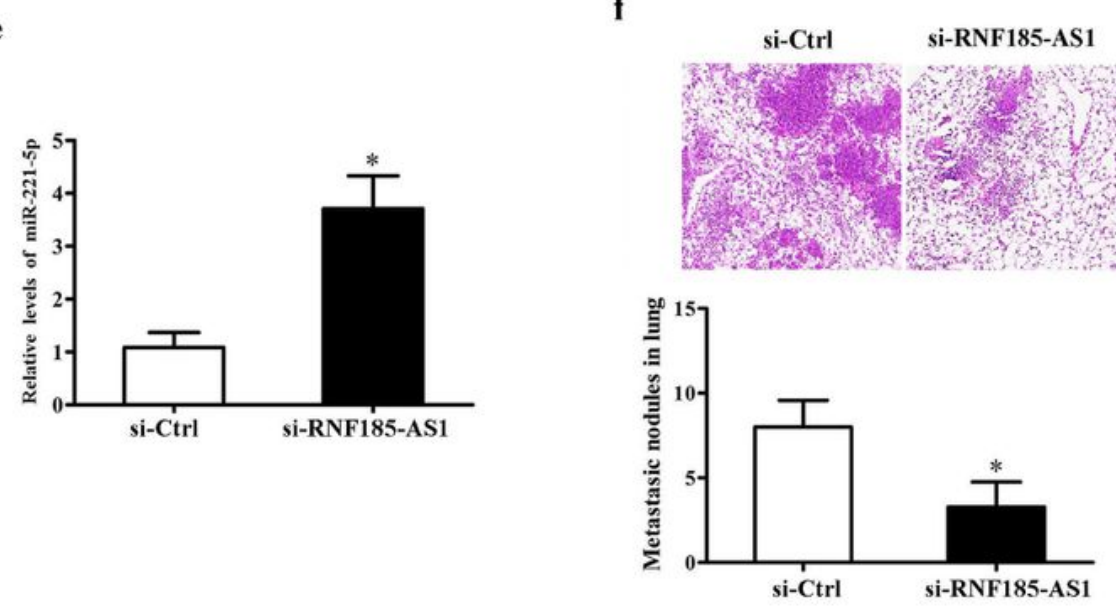

\section{Figure 6}

RNF185-AS1 knockdown inhibited HCC cell metastasis in vivo. Huh7 cells were stably transfected with siRNF185-AS1 or si-Ctrl were subcutaneously injected into nude mice to construct xenograft models. a Representative images of tumors. b, c The volume and weight of tumor xenografts. $d$ Representative immunohistochemical staining of Ki-67, ITGB5, E-cadherin and vimentin. Huh7 cells were stably transfected with siRNF185-AS1 or si-Ctrl were injected to nude mice via the tail vein. e miR-221-5p 
expression were determined by qRT-PCR. $f$ Tumors from mouse xenografts were removed and subjected to H\&E staining and the metastatic nodules in the lung were counted. ${ }^{*} p<0.05$. 